\title{
The effect of consuming red and yellow cherry tomatoes on endothelial function in free-living normotensive males: a randomised cross-over trial
}

\author{
H.M. Cheng ${ }^{1}$, G. Koutsidis ${ }^{1}$, J.K. Lodge ${ }^{1}$, K. Keane ${ }^{1}$, M. Siervo ${ }^{2}$ and J. Lara ${ }^{1}$ \\ ${ }^{1}$ Department of Applied Sciences, Faculty of Health and Life Sciences, University of Northumbria at Newcastle, NE1 \\ 8ST, UK and ${ }^{2}$ Human Nutrition Research Centre, Institute of Cellular Medicine and Newcastle University Institute for \\ Ageing, Newcastle University, Newcastle upon Tyne, UK.
}

Dysfunction of the endothelium has been identified as an early step in the pathogenesis of cardiovascular disease (CVD) $)^{(1)}$. CVDs are the leading cause of death representing $31 \%$ of global deaths ${ }^{(2)}$. Plant-based dietary patterns have been shown to influence cardiovascular risk factors and CVDs. Tomato is a rich source of lycopene, a major carotenoid in human plasma with strong antioxidant properties $^{(3)}$. Previous systematic reviews of randomised controlled trials have shown that supplementing tomato, or lycopene, have positive effects on endothelial function, particularly on improving flow mediated dilation ${ }^{(4)}$; however, its effects on other markers of endothelial function has not been fully investigated. Previous evidence from clinical trials investigating the effects of tomato consumption have focused on studying red tomato, red-tomato products, or lycopene supplementation, however the effect of different tomato varieties has not been explored. With new varieties of tomato available to the public, it is important to evaluate whether these exert the same health effects. The present study investigated whether red cherry tomato (Piccolo variety) characterised by high content of translycopene, and orange cherry tomato (Oranjstar variety) high in cis-lycopene, improves endothelial function to the same extent.

27 healthy males, aged 18-60 years, participated in a randomised, controlled, cross-over, 12-week study investigating the effect of fresh red cherry tomato (Piccolo) compared to orange cherry tomato (Oranjstar). This study included two arm treatments including $300 \mathrm{~g}$ fresh red cherry tomato (Piccolo), orange cherry tomato (Oranjstar) supplementation and a 4-week wash-out period (without tomato consumption). This study was run at the Northumbria University research facilities. Participants were instructed to maintain their dietary and physical activity patterns during the study. Before and after each 4-week intervention, participant attended a study visit for outcome measurements. Pulse wave velocity (PWV) and pulse wave analysis (PWA) were measured using SphygmoCor (CPV System). Flow mediated dilation (FMD) was measured using ultrasound, and microvascular function was measured using laser doppler imaging (LDI; moorLDI2-IR). The study was reviewed by the Northumbria University Ethics Committee (Project ref: PG02_Cheng_121216) and was given a favourable ethical opinion to proceed. This trial was registered in ClinicalTrials.gov (identifier: NCT03209817).

Participants of this study were on average 32 years (SD 14), and had a mean BMI of 24 (SD $3 \cdot 37 \mathrm{~kg} / \mathrm{m}^{2}$ ), resting SBP 116 (SD 11 $\mathrm{mmHg}$ ) and resting DBP $70(\mathrm{SD} 8 \mathrm{mmHg})$ ). Results were evaluated according to age group by classifying participants as $<30$ years $(n=16)$ and $>30$ years $(n=11)$. In the older group, but not in the young group, FMD improved significantly after Piccolo in comparison to Oranjstar tomato consumption (Piccolo mean $7.5 \%$, Orangstar mean $4.1 \%, p=0.026$ ). No significant differences in PWV, PWA, or LDI were observed between treatments, either in the young or the old groups.

In conclusion, consuming $300 \mathrm{~g}$ /day of red cherry tomatoes, but not yellow tomatoes, for 4 weeks significantly improved endothelial function as measured by FMD in healthy adults older than 30 years. Improvements in FMD, independently of other risk factors, are associated with significant reduction in the risk of cardiovascular disease. These results warrant further research on the mechanisms behind the health benefits of tomato consumption.

1. Lara J, Ashor AW et al. (2016) Eur J Nutr 55(2), 451-9.

2. World Health Organization (2016) NCD mortality and morbidity [cited 20164 Aug]. Available from: http://www.who.int/gho/ncd/ mortality_morbidity/en/.

3. Mein JR, Lian F et al. (2008) Nutr Rev 66(12), 667-83.

4. Cheng HM, Koutsidis G et al. (2017) Atherosclerosis 257, 100-8.

5. Cheng HM, Koutsidis G et al. (2017) Crit Rev Food Sci Nutr 1-18. 